$12 ; 12.1$

\title{
Измерения эмиттанса газодинамического электронно-циклотронного резонансного источника ионов
}

\author{
() С.В. Барабин ${ }^{1}$, Г.Н. Кропачев ${ }^{1}$, А.Ю. Лукашин ${ }^{1}$, Т.В. Кулевой ${ }^{1}$, С.С. Выбин ${ }^{2}$, С.В. Голубев ${ }^{2}$, \\ И.В. Изотов ${ }^{2}$, Е.М. Киселева ${ }^{2}$, В.А. Скалыга ${ }^{2,9}$, С.В. Григорьев ${ }^{3}$, Н.А. Коваленко \\ ${ }^{1}$ Институт теоретической и экспериментальной фризики им. А.И. Алиханова Национального исследовательского центра \\ „Курчатовский институт“, Москва, Россия \\ ${ }^{2}$ Институт прикладной физики РАН, Нижний Новгород, Россия \\ ${ }^{3}$ Петербургский институт ядерной физики им. Б.П. Константинова Национального исследовательского центра \\ „Курчатовский институт“, Гатчина, Ленинградская обл., Россия \\ ฯ E-mail: skalyga@ipfran.ru
}

Поступило в Редакцию 24 ноября 2020г.

В окончательной редакции 24 ноября 2020 г.

Принято к публикации 17 февраля 2021г.

\begin{abstract}
Обсуждаются достоинства использования газодинамического источника протонов на основе электронноциклотронного резонанса в компактном нейтронном источнике с протонным ускорителем проекта DARIA. Экспериментально показано, что газодинамический источник протонов обеспечивает генерацию пучка с током $\sim 100 \mathrm{~mA}$, длительностью более $100 \mu \mathrm{s}$ при частоте повторения вплоть до $1000 \mathrm{pulse} / \mathrm{s}$. Методом ,pepperpot $^{\text {“ }}$ измерен эмиттанс пучка, генерируемого газодинамическим источником электронно-циклотронного резонанса с двумя вариантами геометрии электродов формирующей пучок оптики: „сферическими“ и плоскопараллельными. Показано, что нормализованный $4 \mathrm{rms}$-эмиттанс для обоих вариантов электродов системы формирования пучка в диапазоне напряжений экстракции от 41 до $48 \mathrm{kV}$ не превышает $2 \pi \cdot \mathrm{mm} \cdot \mathrm{mrad}$.
\end{abstract}

Ключевые слова: ECR, эмиттанс, pepper-pot, компактный нейтронный источник DARIA.

DOI: 10.21883/PJTF.2021.10.50964.18628

Специально созданная рабочая группа по физической науке и инженерной стратегии (Physical Sciences and Engineering Strategy Working Group, ESFRI) [1] на основе проведенного анализа пришла к выводу, что к 30-м годам текущего столетия число нейтронных пучков, доступных для исследований, будет находиться на уровне $2 / 3$ от доступного на данный момент. Этот уровень будет поддерживаться только при условии, что самый крупный европейский исследовательский реактор ILL в Гренобле продолжит свою работу в максимальном объеме. В качестве альтернативы реакторным источникам нейтронных пучков для научных исследований и промышленного применения все больший интерес представляют „мегасайенс"-установки - нейтронные генераторы на основе высокоинтенсивных протонных ускорителей (IPNS [2], Los Alamos Neutron Science Center [3], ISIS [4], SNS [5], JPARC [6] и ESS [7]). Эти крупные установки - так называемые источники нейтронов на основе реакции испарения-скалывания - способны обеспечить нейтронные потоки, сопоставимые с получаемыми на лучших реакторах. В России разработка прототипа такой установки в ИФВЭ НИЦ „Курчатовский институт“ начата в рамках программы развития синхротронно-нейтронных исследований. Нейтронный генератор IFMIF-EVEDA на базе мощного линейного ускорителя дейтронов и жидколитиевой мишени для исследования материалов, использующихся в реакторах деления, находится на стадии ввода в эксплуатацию в Роккашо (Япония) [8]. Однако проведение обучения специалистов для работы на нейтронных пучках, подготовка нового оборудования, тестирование новых прорывных идей невозможны на таких дорогих установках. Перечисленные выше факторы требуют сети компактных источников различной мощности, которые можно использовать в университетах и научных институтах. По этой причине в дополнение к меганаучным объектам в настоящее время разрабатывается несколько проектов компактных и относительно дешевых источников нейтронов на основе линейных ускорителей ионов. В качестве примера таких проектов можно назвать SARAF в Израиле [9], FRANZ в Германии [10], MUNES в Италии [11]. В России разработка такого источника ведется в рамках проекта DARIA (compact neutron source Dedicated to Academic Research and Industrial Application - компактный нейтронный источник для фундаментальных исследований и промышленных приложений) командой специалистов НИЦ „Курчатовский институт“ из ИТЭФ и ПИЯФ в сотрудничестве с СПбГУ, ОИЯИ, БФУ им. И. Канта и ИПФ РАН $[12,13]$.

Основные элементы компактного источника нейтронов: протонный источник, протонный ускоритель, мишенная сборка и станции нейтронного рассеяния. Цель настоящей работы - экспериментальная проверка и сравнение возможностей двух вариантов работы протонного источника как источника непрерывного и импульсного действия. При выборе параметров компактного источника нейтронов DARIA в качестве ориентира послужил исследовательский реактор BER-II в Берлинском центре материалов и энергии им. Гельмгольца (НZB) (Германия), который обеспечивает пучок нейтронов плотностью $2 \cdot 10^{14} \mathrm{~s}^{-1} \cdot \mathrm{cm}^{-2}$. Для энергии 


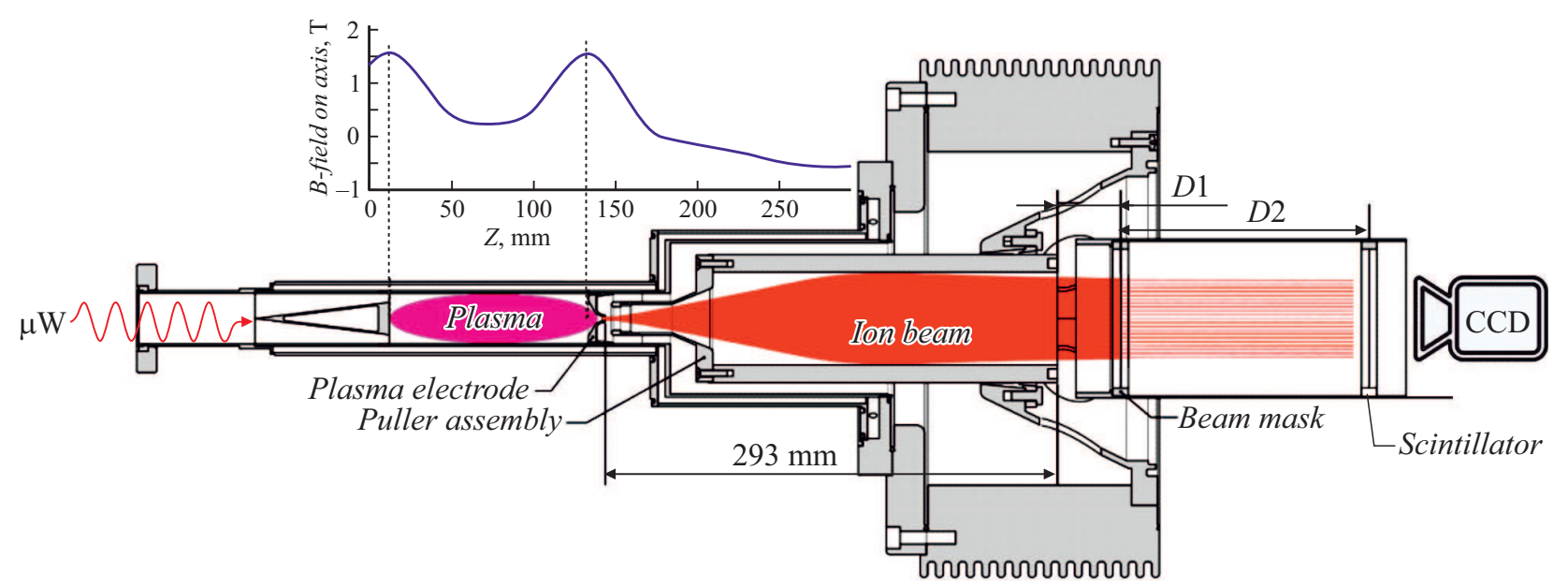

Рис. 1. GISMO ЭЦР-ионный источник и измеритель эмиттанса, установленный на его выходе. Узлы источника и их предназначение описаны в тексте. На вставке представлен профиль магнитного поля в плазменной ловушке.

протонного пучка $13 \mathrm{MeV}$ и бериллиевой мишени была оценена необходимая интенсивность ионного пучка для трех различных режимов генерации нейтронного потока: теплового (длительность импульса $\tau=44 \mathrm{~ms}$ и частота повторения $T=120 \mathrm{~Hz})$, биспектрального $(\tau=66 \mathrm{~ms}$, $T=120 \mathrm{~Hz})$ и холодного $(\tau=110 \mathrm{~ms}, T=20 \mathrm{~Hz})$. Показано, что для указанных режимов на мишень требуется доставить протонный пучок указанной выше энергии с током $80-85 \mathrm{~mA}$. Для решения поставленной задачи рассматриваются два варианта установки. Один вариант, аналогичный реализуемому во Франкфуртском нейтронном источнике в центре Штерна-Герлаха (FRANZ), базируется на линейном ускорителе непрерывного действия (в так называемом $c w$-режиме, continues wave) с последующей компрессией ускоренного пучка относительно невысокой интенсивности [10]. В результате компрессии предполагается формировать на мишени импульсы с током $\sim 100 \mathrm{~mA}$, длительностью $\sim 100 \mu \mathrm{s}$ и регулируемой частотой посыла импульсов вплоть до $1000 \mathrm{~Hz}$. Другой (альтернативный) вариант основан на ускорении высокоинтенсивного импульсного пучка, который изначально обладает приведенными выше параметрами: интенсивностью, длительностью импульса и частотой следования импульсов. Оба варианта имеют свои плюсы и минусы. Вариант с ускорителем непрерывного действия (так называемый $c w$-режим) позволяет ускорять пучки с током на уровне $2-3 \mathrm{~mA}$. Однако при этом требуется решить две сложные физико-технические проблемы: осуществить компрессию ускоренного пучка до требуемых параметров и обеспечить защиту от высокочастотных (ВЧ) пробоев, поскольку в установке, paботающей в непрерывном режиме, это может привести к разрушению электродов. По этой причине предельная напряженность поля на электродах выбирается заметно меньшей, чем для импульсных машин. Как следствие, растут поперечные и продольные размеры ускорителя. Импульсная машина заметно устойчивее к ВЧ-пробоям, однако требуется обеспечить ускорение пучка с интенсивностью на два порядка выше. Результаты разра- ботки варианта ускорителя для работы в непрерывном режиме для проекта DARIA представлены в [12]. Для импульсного варианта предполагается разработать вариант ускорителя под параметры пучка, генерируемого импульсным источником ионов GISMO (Gasdynamic Ion Source for Multipurpose Operation - газодинамический ионный источник для различных приложений), разработанным в ИПФ РАН (Нижний Новгород) [14-18]. Для разработки ускоряющего канала и определения конкурентоспособности импульсного ускорителя проведены измерения эмиттанса данного источника протонов методом „pepper-pot“. Измерения проводились на площадке ИПФ РАН мобильным измерителем эмиттанса, разработанным в ИТЭФ [19]. Для генерации плазмы используется $28 \mathrm{GHz} / 10 \mathrm{~kW}$ гиротрон непрерывного действия, выпускаемый компанией „Гиком“. Схема источника с установленным на его выходе „реpper-pot“-измерителем эмиттанса показана на рис. 1. В источнике используется плазменная ловушка полностью на постоянных магнитах с магнитным полем $1.5 \mathrm{~T}$ в максимуме и $0.25 \mathrm{~T}$ в центре ловушки (зеркальное отношение равно 6). Расстояние между магнитными зеркалами $12 \mathrm{~cm}$. Внутренний диаметр магнитной ловушки $50 \mathrm{~mm}$, что позволяет использовать водоохлаждаемую камеру с внутренним диаметром $32 \mathrm{~mm}$. Ускоряющее пучок напряжение может достигать $100 \mathrm{kV}$. На источнике предусмотрены два варианта электродов для формирования пучка протонов из плазмы электронно-циклотронного резонанса (ЭЦР) разряда. Первый - это набор плоских электродов, второй - система „сферических“ электродов [20].

Измеритель эмиттанса включает медную маску, сцинтиллятор и CCD-камеру. В качестве маски используется медная фольга толщиной $100 \mu \mathrm{m}$ с массивом $20 \times 20$ отверстий, диаметром $0.2 \mathrm{~mm}$ и расстоянием между отверстиями $2.5 \mathrm{~mm}$. Сцинтиллятор - кристалл CsI диаметром $100 \mathrm{~mm}$. Маска и сцинтиллятор расположены в общей оправке, монтирующейся внутри измерительной камеры ионного источника. Для измерений использовалась стробируемая видеокамера На- 

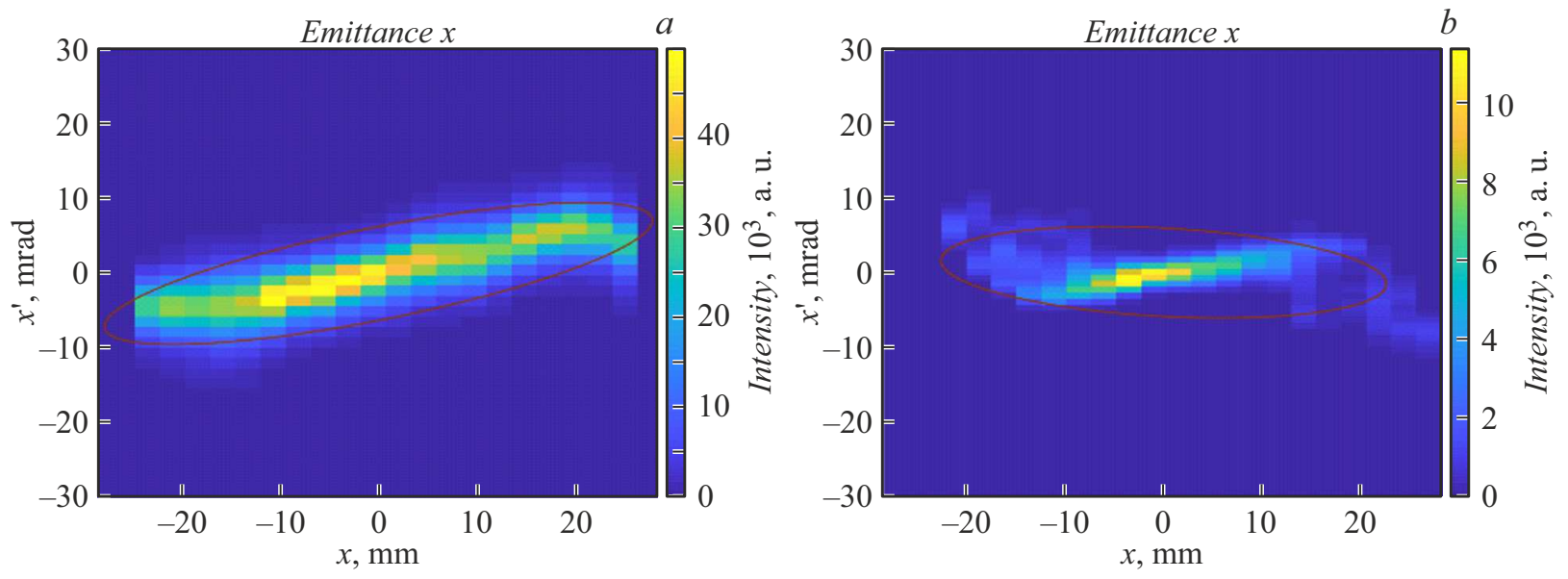

Рис. 2. Интенсивность пучка на фазовой плоскости $x-x^{\prime}$ для измерений со „сферическим“ $(a)$ и плоским $(b)$ экстракторами. Значение $4 \mathrm{rms}$-эмиттанса для „сферической“ конфигурации электродов равно $1.71 \mathrm{\pi} \cdot \mathrm{mm} \cdot \mathrm{mrad}$, для плоскопараллельных электродов $-1.31 \pi \cdot \mathrm{mm} \cdot \mathrm{mrad}$.
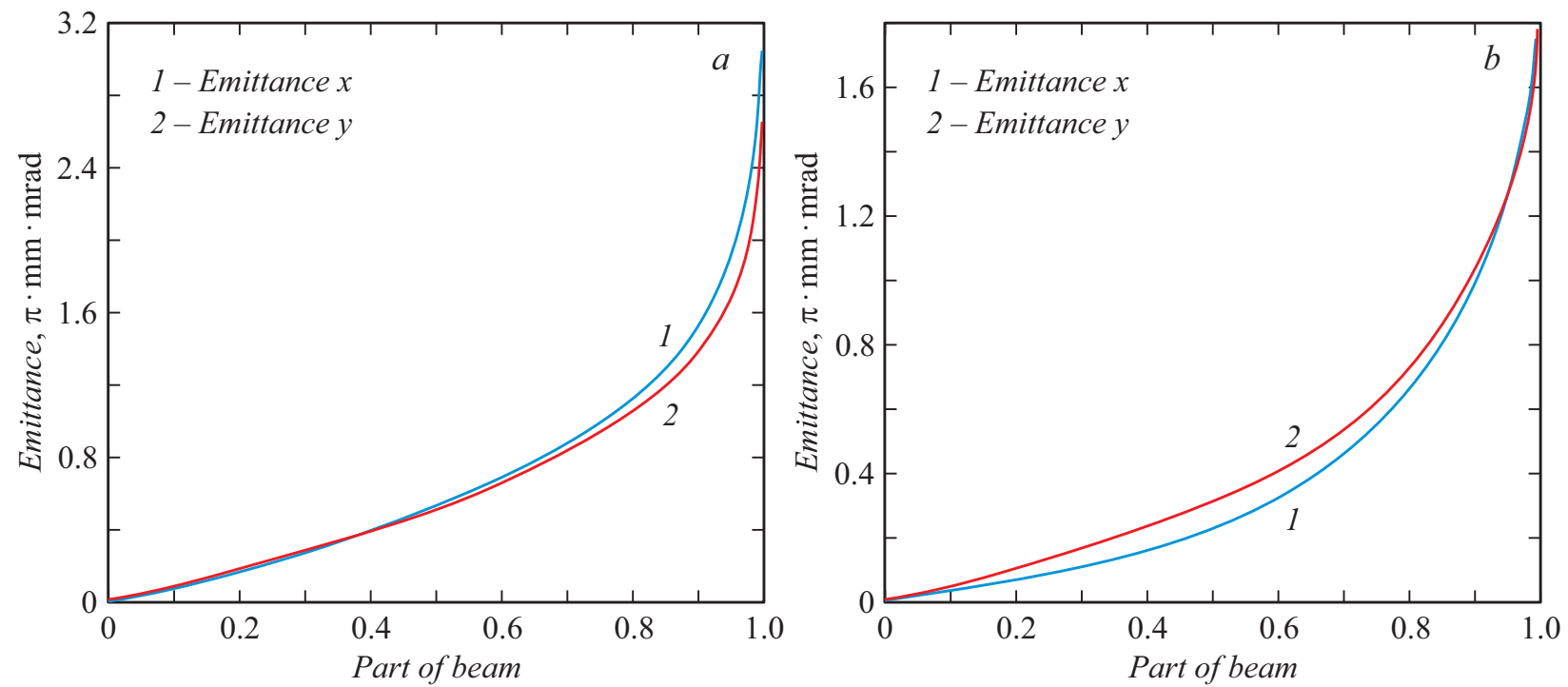

Рис. 3. Зависимости значений нормализованных эмиттансов от доли тока пучка для „сферического“ $(a)$ и плоского $(b)$ экстракторов.

ногейт 24-3 от НПП „Наноскан“ с CCD-матрицей разрешением $1380 \times 1040$ пикселей, выходные сигналы с которой оцифровываются 12-разрядными аналогоцифровыми преобразователями, длительность экспозиции видеокамеры в ходе экспериментов равнялась $20 \mu \mathrm{s}$. Объектив видеокамеры располагался в кожухе для защиты CCD-матрицы от внешней засветки [6].

В ходе эксперимента было проведено две серии измерений: со „сферическими“ электродами (пуллер на рис. 1) и с плоскопараллельными плазменным и вытягивающим электродами. Эксперименты проводились при давлении в разрядной камере 2 mTorr при двух значениях мощности гиротрона: 2.8 и $4.5 \mathrm{~kW}$.

В ходе экспериментов со „сферической“ конфигурацией электродов для каждого из значений мощности были проведены серии измерений с напряжениями экстракции в диапазоне от 35 до $51 \mathrm{kV}$. Необходимо отметить, что с увеличением напряжения экстракции ток пучка на маску увеличивался с 55 до $100 \mathrm{~mA}$. Маска располагалась в положении $D 1=101 \mathrm{~mm}$ (рис. 1), расстояние между маской и сцинтиллятором CsI $D 2=80 \mathrm{~mm}$.

При экспериментах с плоскопараллельными электродами для каждого из значений мощности были проведены серии измерений с напряжениями экстракции в диапазоне от 35 до $48 \mathrm{kV}$. Ток пучка на маску увеличивался с увеличением напряжения экстракции от 52 до $90 \mathrm{~mA}$. В ходе измерений $D 1=44 \mathrm{~mm}$, а $D 2=137 \mathrm{~mm}$.

На рис. 2 приведены изображения пучка на фазовой плоскости $x-x^{\prime}$ для измерений со „сферическим“ и плоским экстракторами. На рисунках представлены эллипсы, соответствующие 4 rms-эмиттансу пучка, определенному по результатам обработки полученных данных. На рис. 3 показаны зависимости эмиттансов от доли тока пучка для „сферической“ и плоской геометрии электро- 
дов. Как видно из полученного результата, эмиттансы пучка для двух вариантов электродов заметно различаются как по величине, так и по распределению фазовой плотности. Для варианта со „сферической“ геометрией пучок равномерно заполняет эллипс $4 \mathrm{rms}$-эмиттанса, в то время как для плоскопараллельных электродов распределение имеет ядро и $S$-образные хвосты, что свидетельствует о большом влиянии нелинейных эффектов на формирование пучка. Однако для варианта с плоскопараллельными электродами, используя набор диафрагм, при достаточной интенсивности пучка можно сформировать пучок с заметно меньшим эмиттансом при более высокой фазовой плотности.

В ходе измерений было показано, что под влиянием магнитного поля источника, простирающегося в область формирования пучка (рис. 1), пучок являлся сходящимся для малых напряжений экстракции и расходящимся для больших. Кроссовер пучка в точке проведения измерения получается при ускоряющем напряжении $\sim 44 \mathrm{kV}$.

В заключение отметим следующее: проведенные измерения показали, что нормализованный 4 rms-эмиттанс для обоих вариантов электродов системы формирования пучка в диапазоне напряжений экстракции от 41 до $48 \mathrm{kV}$ не превышает $2 \pi \cdot \mathrm{mm} \cdot \mathrm{mrad}$. Данное значение эмиттанса принято за базовое для разработки ускоряющего канала импульсного линейного ускорителя. Экспериментально показано, что газодинамический источник протонов обеспечивает генерацию пучка с током порядка $100 \mathrm{~mA}$, длительностью более $100 \mu \mathrm{s}$ при частоте повторения вплоть до 1000 pulse/s, а следовательно, такой источник протонов может быть успешно использован на компактных (с малым и средним потоком) импульсных источниках нейтронов на основе ускорителей. По результатам разработки будет проведен сравнительный анализ с ускоряющей структурой $c w$-ускорителя, на основании которого будет выбран вариант для создания рабочего образца компактного источника нейтронов на основе линейного ускорителя протонов.

\section{Финансирование работы}

Разработка источника и тестового стенда выполнена в рамках государственного задания № 0035-2019-0002 и гранта Президента РФ № МД-2745.2019.2. Измерение эмиттанса ионного пучка проведено при поддержке гранта Российского научного фонда № 19-12-00363.

\section{Конфликт интересов}

Авторы заявляют, что у них нет конфликта интересов.

\section{Список литературы}

[1] Neutron scattering facilities in Europe: present status and future perspectives (ESFRI Scripta, 2016), vol. 1.

[2] IPSN [Электронный ресурс]. Режим доступа: www.aai.anl.gov/history/project_pages/ipns.html
[3] LANSCE [Электронный ресурс]. Режим доступа: http://lansce.lanl.gov/

[4] ISIS [Электронный ресурс]. Режим доступа: http://www.isis.stfc.ac.uk/

[5] SNS [Электронный ресурс]. Режим доступа: http://neutrons.ornl.gov/sns

[6] JPARC [Электронный ресурс]. Режим доступа: http://j-parc.jp/index-e.html

[7] ESS [Электронный ресурс]. Режим доступа: http://europeanspallationsource.se/

[8] E. Fagotti, L. Antoniazzi, L. Bellan, D. Bortolato, M. Comunian, A. Facco, M.G. Giacchini, F. Grespan, M. Montis, A. Palmieri, A. Pisent, F. Scantamburlo, B. Bolzon, N. Chauvin, R. Gobin, P. Cara, H. Dzitko, D. Gex, A. Jokinen, G. Phillips, T. Ebisawa, A. Kasugai, K. Kondo, K. Sakamoto, T. Shinya, M. Sugimoto, R. Heidinger, A. Marqueta, I. Moya, P. Mereu, G. Pruneri, M. Weber, in Proc. Int. Particle Accelerator Conf (IPAC 2018) (Vancouver, Canada, 2018), p. 2902. DOI: 10.18429/JACoW-IPAC2018-THXGBF2

[9] N. Pichoff, D. Chirpaz-Cerbatpresenter, R. Cubizolles, J. Dumas, R.D. Duperrier, G. Ferrand, B. Gastineau, P. Gastinel, F. Gougnaud, M. Jacquemet, C. Madec, Th. Plaisant, F. Senée, A. Sutra-Fourcade, D. Uriot, D. Berkovits, J. Luner, A. Perry, E. Reinfeld, J. Rodnizki, M. Di Giacomo, in Proc. Int. Particle Accelerator Conf. (IPAC 2018) (Vancouver, Canada, 2018), p. 994. DOI: 10.18429/JACoW-IPAC2018-TUPAK015

[10] C. Wiesner, S.M. Alzubaidi, M. Droba, M. Heilmann, O. Hinrichs, B. Klump, O. Meusel, D. Noll, O. Payir, H. Podlech, U. Ratzinger, A. Schempp, S. Schmidt, P.P. Schneider, M. Schwarz, W. Schweizer, K. Volk, C. Wagner, R. Reifarth, in Proc. Int. Particle Accelerator Conf. (IPAC 2015) (Richmond, USA, 2015), p. 1276. DOI: 10.18429/JACoW-IPAC2015-TUXB

[11] A. Pisent, E. Fagotti, P. Colautti, in Proc. Linear Accelerator Conf. (LINAC 2014) (Geneva, Switzerland, 2014), p. 261. DOI: 10.18429/JACoW-LINAC2014-MOPP088

[12] G. Kropachev, T. Kulevoy, A. Sitnikov, J. Synch. Investig., 13 (6), 1126 (2019). DOI: 10.1134/S1027451019060399

[13] S. Grigoriev, E. Iashina, K. Pavlov, J. Synch. Investig., 13 (6), 1132 (2019). DOI: 10.1134/S1027451019060314

[14] S. Golubev, I. Izotov, S. Razin, A. Sidorov, V. Skalyga, A. Vodopyanov, V. Zorin, A. Bokhanov, Nucl. Instrum. Meth. Phys. Res. B, 256 (1), 537 (2007).

[15] V. Skalyga, I. Izotov, S. Golubev, A. Sidorov, S. Razin, A. Vodopyanov, O. Tarvainen, H. Koivisto, T. Kalvas, Rev. Sci. Instrum., 87 (2), 02A716 (2016).

[16] V.A. Skalyga, I.V. Izotov, A.V. Sidorov, S.V. Golubev, S.V. Razin, Rev. Sci. Instrum., 88 (3), 033503 (2017).

[17] V.A. Skalyga, I.V. Izotov, S.V. Golubev, S.V. Razin, A.F. Bokhanov, M.Y. Kazakov, R.L. Lapin, R.A. Shaposhnikov, E.A. Mironov, A.V. Voitovich, O.V. Palashov, G.G. Denisov, V.I. Belousov, D.I. Sobolev, M.Y. Shmelev, M.Y. Glyavin, A.I. Tsvetkov, M.V. Morozkin, M.D. Proyavin, AIP Conf. Proc., 2011, 030013 (2018).

[18] V. Skalyga, I. Izotov, S. Golubev, A. Sidorov, S. Razin, A. Strelkov, O. Tarvainen, H. Koivisto, T. Kalvas, J. Appl. Phys., 118 (9), 093301 (2015).

[19] S. Barabin, A.V. Kozlov, T. Kulevoy, D.A. Liakin, A.Y. Lukashin, D.N. Selesnev, in Proc. 26th Russian Particle Accelerator Conf. (RUPAC'18) (Protvino, Russia, 2018), paper THPSC18, p. 443. DOI: 10.18429/JACoW-RUPAC2018-THPSC18

[20] S.S. Vybin, I.V. Izotov, V.A. Skalyga, Plasma Sources Sci. Technol., 29 (11), 11LT02 (2020). https://doi.org/10.1088/1361-6595/abbf9c 